\title{
O espaço de trabalho da enfermagem em Unidades Neonatal e os riscos laborais:
}

\section{revisão integrativa}

The work space of nursing in Neonatal Units and occupational risks: integrative review

El espacio de trabajo de enfermería en las Unidades Neonatales y los riesgos laborales: una revisión integradora

Ana Patrícia Batista Silva

ORCID: https://orcid.org/0000-0002-2537-0388

Universidade Federal de Goiás, Brasil

E-mail: anapatybs@outlook.com

Marinésia Aparecida do Prado

ORCID: https://orcid.org/0000-0003-4652-0733

Universidade Federal de Goiás, Brasil

E-mail: marinesiaprado@gmail.com

Ludimila Cristina Souza Silva

ORCID: https://orcid.org/0000-0002-4342-217X

Centro Universitário Alfredo Nasser, Brasil

E-mail: dra.ludimilacristina@gmail.com

Lorena Zenha Andrade

ORCID: https://orcid.org/0000-0002-1065-7917

Centro Universitário Alfredo Nasser, Brasil

E-mail: lorenazenha@hotmail.com

Henrique Pascoa

ORCID: https://orcid.org/0000-0003-2542-6879

Centro Universitário Alfredo Nasser, Brasi

E-mail: hpascoa2@gmail.com

Lydia Raquel Rodrigues Bastos

ORCID: https://orcid.org/0000-0002-2540-8016

Faculdade Noroeste, Brasil

E-mail: lydiaraque12015@gmail.com

Kelly Cristina Pereira dos Santos

ORCID: https://orcid.org/0000-0002-4447-860X

Faculdade Noroeste, Brasil

E-mail: enfermeirakelly2@gmail.com

Paulo Sérgio Santos Junior

ORCID: https://orcid.org/0000-0001-6399-0978 Faculdade Noroeste, Brasil

E-mail: paulo--junior@hotmail.com

Ellen Halitta Arantes

ORCID: https://orcid.org/0000-0002-3208-5454 Faculdade Padrão, Brasil

E-mail: ellenhalitta@gmail.com

Jessica da Silva Campos

ORCID: https://orcid.org/0000-0002-6254-7250 Universidade Paulista, Brasil

E-mail: jsilvacampos18@gmail.com

Júlio César Coelho do Nascimento

ORCID: https://orcid.org/0000-0002-1783-842X

Faculdade Noroeste, Goiânia-GO, Brasil

E-mail: prof.julioccnascimento@gmail.com

Maria Alves Barbosa

ORCID: https://orcid.org/0000-0002-0861-9655 Universidade Federal de Goiás, Brasil

E-mail: maria.malves@gmail.com

Marislei Espindula Brasileiro

ORCID: https://orcid.org/0000-0003-3594-6384 Faculdade Unida de Campinas, Brasil

E-mail: marislei@cultura.trd.br

Ana Lucia Queiroz Bezerra

ORCID: https://orcid.org/0000-0002-3330-8859

Universidade Federal de Goiás, Brasil

E-mail: analuciaqueiroz@uol.com.br 


\begin{abstract}
Resumo
Objetivou-se levantar, na literatura nacional e internacional, as evidências científicas acerca dos indicadores de riscos ocupacionais e os reflexos destes para a saúde da equipe de enfermagem em unidade neonatal. Estudo de revisão integrativa, realizado durante o mês de outubro de 2020. A busca foi realizada por meio do portal da Biblioteca Virtual em Saúde, nas bases de dados LILACS, MEDLINE, IBECS e BDENF, de forma simultânea, por artigos em inglês, português e espanhol com resumo e texto disponíveis na íntegra, publicados entre 2015 e 2020. Excluíram-se os artigos em duplicata, os de revisão, os classificados metodologicamente como tese, dissertação ou monografia bem como aqueles que não se enquadravam no tema. Identificou-se 15 publicações, das quais, 5 atenderam os critérios de elegibilidade. Quanto ao risco ocupacional identificou-se: dano psicológico: estresse ocupacional; burnout; desejo de abandonar o trabalho, exaustão emocional, desmotivação profissional; dano físico: sobrecarga de trabalho; condições ambientais inadequadas e ruído; dano social: dificuldades de relacionamento interpessoal e necessidade de restringir os contatos pessoais. Os reflexos na saúde do trabalhador foram: depressão, frustração, raiva, amargura, negatividade e compulsividade; fadiga, exaustão, falta de atenção, baixa imunidade; irritabilidade, cinismo, baixa empatia. Os riscos laborais refletem negativamente na saúde dos trabalhadores de enfermagem. Recomenda-se estratégias de prevenção e controle destes, buscando promover um ambiente laboral em conformidade com os princípios da segurança vigente na legislação.
\end{abstract}

Palavras-chave: Enfermagem; Riscos ocupacionais; Saúde do Trabalhador; Terapia Intensiva Neonatal.

\begin{abstract}
The objective was to raise, in national and international literature, as scientific evidence about occupational risk indicators and their reflexes for the health of the nursing team in a neonatal unit. Integrative review study, carried out during the month of October 2020. A search conducted through the Virtual Health Library portal, in the LILACS, MEDLINE, IBECS and BDENF databases, simultaneously, for articles in English, Portuguese and Spanish with abstract and full text available, published between 2015 and 2020. Duplicate articles, review, methodologically classified as thesis, dissertation or monograph as well as those that did not fit the theme were excluded. 15 publications were identified, of which, 5 met the eligibility criteria. As for occupational risk, it was identified: psychological damage: occupational stress; exhaustion; desire to quit work, emotional exhaustion, professional demotivation; physical damage: work overload; inadequate environmental conditions and noise; social damage: difficulties in interpersonal relationships and the need to restrict personal contacts. The reflexes on the worker's health were: depression, frustration, anger, bitterness, negativity and compulsiveness; fatigue, exhaustion, lack of attention, low immunity; irritability, cynicism, low empathy. Occupational risks reflect negatively on the health of nursing workers. The strategy of prevention and control of these is recommended, seeking to promote a working environment in accordance with the principles of safety in force in the legislation.
\end{abstract}

Keywords: Nursing; Occupational risks; Worker's health; Neonatal Intensive Care.

\title{
Resumen
}

El objetivo fue levantar, en la literatura nacional e internacional, la evidencia científica sobre los indicadores de riesgo ocupacional y sus consecuencias para la salud del equipo de enfermería en una unidad neonatal. Estudio de revisión integrativa, realizado durante el mes de octubre de 2020. La búsqueda se realizó a través del portal de la Biblioteca Virtual en Salud, en las bases de datos LILACS, MEDLINE, IBECS y BDENF, simultáneamente, de artículos en inglés, portugués y español con resumen y texto completo. disponibles, publicados entre 2015 y 2020. Se excluyeron artículos duplicados, artículos de revisión, aquellos clasificados metodológicamente como tesis, disertación o monografía, así como aquellos que no encajaran en la temática. Se identificaron 15 publicaciones, de las cuales 5 cumplieron los criterios de elegibilidad. En cuanto al riesgo ocupacional, se identificó: daño psicológico: estrés ocupacional; agotamiento deseo de dejar el trabajo, agotamiento emocional, desmotivación profesional; daño físico: sobrecarga de trabajo; condiciones ambientales inadecuadas y ruido; daño social: dificultades en las relaciones interpersonales y necesidad de restringir los contactos personales. Los efectos sobre la salud de los trabajadores fueron: depresión, frustración, enfado, amargura, negatividad y compulsividad; fatiga, agotamiento, falta de atención, baja inmunidad; irritabilidad, cinismo, baja empatía. Los riesgos laborales repercuten negativamente en la salud de los trabajadores de enfermería. Se recomiendan estrategias de prevención y control, buscando promover un ambiente de trabajo acorde con los principios de seguridad vigentes en la legislación.

Palabras clave: Enfermería; Riesgos laborales; Salud del trabajador; Cuidados intensivos neonatales.

\section{Introdução}

As inovações tecnológicas, advindas da expansão industrial, marcam a evolução da sociedade, e são responsáveis pelas modificações nas relações de trabalho que se constituem na atualidade (Pires et al., 2012). Aliado a esse processo surgem novas formas de adoecimento, resultado do desequilíbrio entre as necessidades do indivíduo e as exigências da organização do trabalho no espaço laboral (Dejours, 2011; Mendes, 2007). 
Desta forma, mediante as condições em que é executado, o trabalho passa a exercer um caráter danoso sobre à saúde dos trabalhadores (Agostini, 2002; Maciel, Santos \& Rodrigues, 2015). Destaque especial merece o âmbito hospitalar, tendo em vista a sua tipicidade insalubre capaz de produzir danos à saúde humana, que vão desde o desgaste físico e psicológico, até a deterioração dos vínculos sociais dos trabalhadores (Forte et al., 2014).

Inserido no contexto hospitalar, as unidades neonatais, ganham destaque por suas características peculiares, tais como: alta complexidade e especificidade, presença de equipamentos de tecnologia avançada, convivência constante com o limiar vida-morte, alta rotatividade, ritmo intenso de trabalho, dentre outros (Nunes \&Tomas, 2013; Oliveira et al., 2013). A existência desses elementos prediz, constantemente, a ocorrência de danos entre os trabalhadores aí inseridos (Benedett, Ferraz \& Posso, 2009; Pedroza, Campos \& Oliveira, 2006; Rolim, Oliveira \& Cardoso, 2003).

Particularmente, a equipe de enfermagem desponta-se como a categoria que representa a maior força de trabalho dentro desses espaços, ocupando uma posição singular (Carvalho et al., 2017). E, por estar em contato direto e por mais tempo com os pacientes, são, também, mais afetados pelos diferentes fatores que predispõem a ocorrência do dano ocupacional (Ribeiro \& Shimizu, 2007).

Entre esses fatores estão inclusas as condições adversas de trabalho, representadas por déficit de recursos humanos e materiais; mobiliário inadequado; carga horária elevada; trabalho em turnos; trabalho noturno; ritmo acelerado e as próprias atribuições realizadas no cotidiano (Masson, Brito \& Athayde, 2011; Terra et al., 2011). Além de uma política precária envolvendo o processo de trabalho, o que torna os trabalhadores vulneráveis aos riscos laborais (Costa et al., 2013; Karino, Martins \& Bobroff, 2011).

A exposição a estes fatores viabiliza a concretização do dano, e, por conseguinte, de inúmeras consequências. Tais como: desequilíbrio físico, mental e social, associadas ao estresse ocupacional, que contribui para o afastamento por licença médica, e para elevar os índices de absenteísmos, gerando sobrecarga naqueles que permanecem, criando, assim, um círculo vicioso (Aytekin, Yilmaz \& Kuguoglu, 2013; Braithwaite, 2008).

Ante ao paradoxo que se institui, dado que cuidar de enfermos permite adoecerem as pessoas que deles cuidam, e ao impacto que a iminência do dano prevê (Ribeiro \& Shimizu, 2007), é urgente a realização de estudos que visem compreender os riscos associados ao trabalho no intuito de reduzir os agravos e promover à saúde dos trabalhadores de enfermagem, em especial os que atuam em unidade neonatal.

Diante disto, e por entender que ainda há pouca literatura que versa sobre essa temática, emergiu o interesse por desenvolver um estudo de revisão integrativa. Esta se justifica, por se tratar de um método que possibilita o conhecimento e interpretação da produção científica, com vistas a acrescer bases para formulação de futuras investigações, fundamentadas em evidências. Assim, elucidou-se como objetivo desse estudo, levantar as evidências científicas acerca dos indicadores de riscos ocupacionais e os reflexos destes para a saúde da equipe de enfermagem em unidades neonatal.

\section{Metodologia}

Trata-se de uma revisão integrativa da literatura, realizada durante o mês de outubro de 2020 e revisada no mês de setembro de 2021. Este tipo de estudo, reúne achados de estudos desenvolvidos mediante diferentes metodologias, sobre um delimitado tema ou questão, de maneira sistemática e ordenada, contribuindo para o aprofundamento do conhecimento do tema investigado (Ercole, Melo \& Alcoforado, 2014). Devido à essas características, têm o potencial de desempenhar um importante papel na Prática Baseada em Evidências (PBE) da Enfermagem (Mendes, Silveira, Galvão \& 2008).

Para a construção da revisão integrativa faz-se necessário o seguimento de fases que contemplem seu rigor metodológico. Tais fases foram adotadas neste estudo e incluíram as seguintes etapas: a) identificação do tema e formulação da questão da pesquisa; b) estabelecimento de critérios de inclusão e exclusão dos estudos para amostragem; c) coleta dos dados 
que serão extraídos dos estudos; d) análise crítica dos estudos selecionados; e) interpretação dos resultados; f) apresentação da síntese estabelecida e revisão dos conteúdos (Souza, Silva \& Carvalho, 2010).

A pergunta norteadora da pesquisa foi formulada de acordo com a estratégia PICO. Nessa perspectiva, definiu-se como questão: Quais são as evidências científicas acerca dos indicadores de riscos ocupacionais e os reflexos destes para a saúde da equipe de enfermagem em unidades neonatal? Posteriormente, delinearam-se como descritores, para estratégia de busca dos estudos, os Descritores em Ciências da Saúde (DeCS) e Medical Subject Headings (MeSH): "Occupational Exposure"; "Neonatal Nursing" e "Intensive Care Units, Neonatal", os quais foram combinados mediante o uso dos operadores booleanos.

A busca da literatura foi realizada pelo portal da Biblioteca Virtual de Saúde (BVS) por meio das seguintes bases de dados: Literatura Latino-Americana e do Caribe em Ciências da Saúde (LILACS), Medical Literature Analysis and Retrieval System on-line (MEDLINE), BDENF (Banco de dados de Enfermagem) e IBECS (Índice Bibliográfico Español en Ciencias de la Salud). Para seleção dos estudos foram estabelecidos como critérios de elegibilidade: artigos originais, publicados nos idiomas inglês, português e espanhol, dentro do recorte temporal de 2015 a 2020, com resumo e texto disponíveis na íntegra. Excluíram-se os artigos em duplicata, os de revisão, os classificados metodologicamente como tese, dissertação ou monografia bem como aqueles que não se enquadravam no tema. O levantamento foi realizado simultaneamente nas bases de dados.

A consulta às bases de dados, mediante a combinação dos descritores, retornou 15 publicações, dos quais, após a primeira seleção, feita pela leitura preliminar do título e aplicação dos critérios estabelecidos, restringiram-se em 6. Realizouse a leitura na íntegra dos títulos e resumos dos artigos, elegíveis para esta etapa. Foi excluído 1 estudo por não abordar o tema investigado, sendo a amostra, constituída de 5 publicações (Figura 1).

Figura 1 - Fluxograma PRISMA de seleção dos estudos para revisão integrativa. Goiânia, GO, 2021.

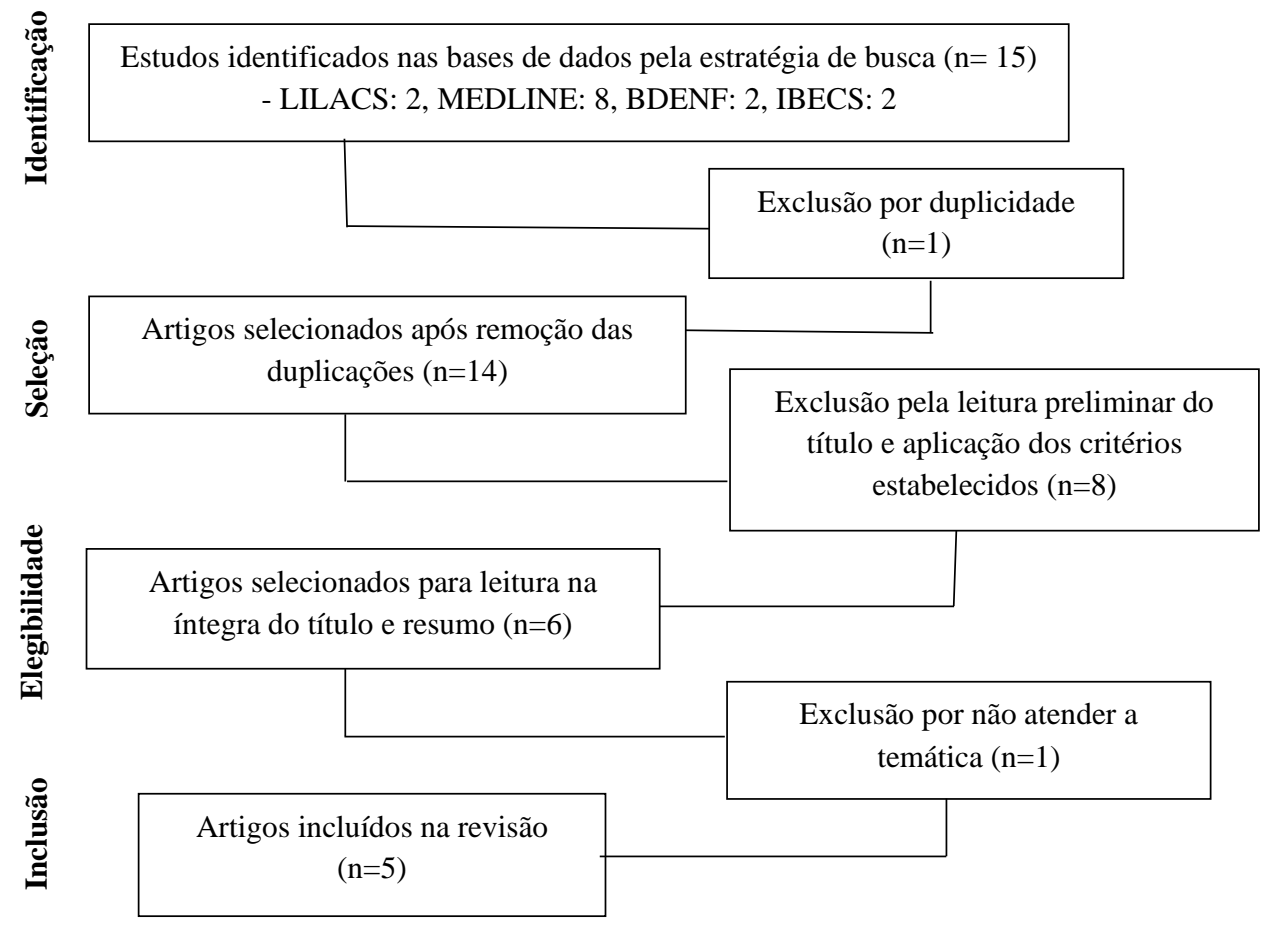

Fonte: Fluxograma PRISMA, adaptado (2021). 
Para a coleta de dados, utilizou-se um instrumento proposto por Ursi (2005), adaptado para esta pesquisa, que abrangeu os seguintes itens: identificação do artigo, características e avaliação do rigor metodológico, intervenções efetivadas e resultados obtidos.

Após leitura criteriosa dos artigos, estes foram classificados e analisados em conformidade com os níveis de evidência classificados por Melnyk e Fineout-Overholt (2005), em sete níveis: nível I, evidências de revisão sistemática ou metanálise de ensaios clínicos randomizados, controlados ou oriundas de diretrizes clínicas; nível II, evidências de pelo menos um ensaio clínico randomizado controlado bem delineado; nível III, evidências de ensaios clínicos bem delineados, não randomizado; nível IV, evidências de estudos de coorte e de caso-controle, bem delineados; nível V, evidências de revisão sistemática de estudos descritivos e qualitativos; nível VI, evidências de um único estudo descritivo ou qualitativo; nível VII, evidências de opinião de autoridades e/ou relatório de comitês de especialistas. As evidências pertencentes aos níveis I e II são consideradas fortes, de III a V evidências moderadas e VI e VII evidências fracas.

A análise crítica das publicações selecionadas foi considerada e tratada de forma descritiva, quanti-qualitativa e os resultados apresentados seguiram o rigor e recomendações da ferramenta PRISMA (Preferred Reporting Items for Systematic Reviews and Meta-Analysis). Esta fase foi disposta em dois momentos, no primeiro, buscou-se caracterizar os estudos e adotou-se quadros sinópticos para sumarização das informações, que contemplaram: título da publicação, autor (es), nome do periódico, ano e país de publicação. No segundo, os resultados dos respectivos níveis de evidência de cada estudo. Cada estudo foi codificado por um número e exposto em ordem crescente, quanto ao ano de publicação.

Em seguida, foram descritos os indicadores de riscos relacionados à prática da enfermagem no contexto neonatal. Os achados foram agrupados por semelhança e organizados em duas categorias temáticas: fatores que predispõe o dano ocupacional no âmbito neonatal e principais consequências relacionadas à ocorrência do dano no ambiente laboral, que foram discutidas à luz do referencial teórico adotado e da literatura pertinente ao tema.

\section{Resultados e Discussão}

\section{Caracterização dos estudos}

Das 5 (100\%) publicações selecionadas, duas (40\%) estavam indexadas na base de dados da MEDLINE, duas (40\%) no IBECS, e uma (20\%) estava indexada tanto na LILACS quanto BDENF. A caracterização dos estudos, segundo título, autor (es), nome do periódico, ano e país de publicação está sintetizada na Tabela 1. 
Tabela 1 - Categorização dos estudos (n=5) quanto ao título, autor(es), nome do periódico, ano e país de publicação. Goiânia, GO, 2020.

\begin{tabular}{|c|c|c|c|c|c|}
\hline $\mathbf{N}^{\mathbf{0}}$ & Título & Autor (es) & Periódico & Ano & País \\
\hline 01 & News of the Academy of Neonatal Nursing & Curtis et al & Neonatal Network & 2015 & USA \\
\hline 02 & $\begin{array}{l}\text { Psychosocial factors and mental work load: a } \\
\text { reality perceived by nurses in intensive care units }\end{array}$ & $\begin{array}{c}\text { Ceballos-Vásquez et } \\
\text { al }\end{array}$ & $\begin{array}{l}\text { Rev. Latino-Am. } \\
\text { Enfermagem }\end{array}$ & 2015 & Chile \\
\hline 03 & $\begin{array}{l}\text { Nivel continuo equivalente de ruido en la } \\
\text { unidad de cuidado intensivo neonatal asociado } \\
\text { al síndrome de burnout. }\end{array}$ & $\begin{array}{l}\text { Galindo;Caicedo \& } \\
\text { Vélez-Pereira }\end{array}$ & $\begin{array}{l}\text { Enferm } \\
\text { Intensiva }\end{array}$ & 2015 & Colombia \\
\hline 04 & $\begin{array}{l}\text { Striving for Optimum Noise-Decreasing } \\
\text { Strategies in Critical Care: Initial } \\
\text { Measurements and Observations. }\end{array}$ & Disher et al & $\begin{array}{l}\text { J Perinat Neonatal } \\
\text { Nurs }\end{array}$ & 2017 & Canadá \\
\hline 05 & $\begin{array}{l}\text { Estudio descriptivo sobre las condiciones de } \\
\text { trabajo y los trastornos musculo esqueléticos } \\
\text { en el personal de enfermería (enfermeras y } \\
\text { AAEE) de la Unidad de Cuidados Intensivos } \\
\text { Pediátricos y Neonatales en el Hospital Clínico } \\
\text { Universitario de Valladolid }\end{array}$ & Rizo;Ubago & Med. segur. trab & 2018 & Espanha \\
\hline
\end{tabular}

Fonte: Os autores.

No que tange ao recorte temporal, constatou-se escassez de estudos referentes a temática, haja vista que no ano de 2015, identificou-se apenas três (60\%) publicações, e em 2017 e 2018 uma (20\%) em cada. Nesta perspectiva, verificou-se ainda ausência de estudos brasileiros que discorressem sobre o tema abordado. Estudos internacionais representaram a totalidade das publicações, dos quais um (20\%) foi realizado na Colômbia e um (20\%) na Espanha, ambos publicados no idioma espanhol. Três (60\%) foram publicados em inglês, e desenvolvidos nos Estados Unidos, Canadá e Chile.

Em se tratando do tipo de metodologia aplicada aos estudos, identificou-se predomínio da pesquisa descritiva, três (60\%), seguidas do estudo de coorte, um (20\%) e relatório de comitê de especialistas, um (20\%). Baseado nesta análise procedeu-se a classificação do nível de evidência dos artigos, dos quais, a maioria, três (60\%), classificaram-se com nível de evidência 6, um (20\%) com nível 7 e um (20\%) com nível 4 (Tabela 2).

Tabela 2 - Classificação do nível de evidência dos estudos analisados. Goiânia, GO, 2020.

\begin{tabular}{llc}
\hline $\mathbf{N}^{\mathbf{0}}$ & \multicolumn{1}{c}{ Delineamento metodológico } & Nível de evidência \\
\hline 01 & Relatório de Comitê de Especialistas & VII \\
02 & Estudo quantitativo, descritivo, transversal & VI \\
03 & Estudo de coorte & IV \\
04 & Estudo quantitativo, descritivo & VI \\
05 & Estudo quantitativo, descritivo, transversal & VI \\
\hline
\end{tabular}

Fonte: Os autores.

Quanto as análises dos objetivos dos estudos, verificou-se que um (5) tratou das condições de trabalho, estresse ocupacional, distúrbios musculoesqueléticos e síndrome de burnout em enfermeiros de unidades neonatal; três (1, 3 e 4) mensuraram o nível de ruído em Unidade de Terapia Intensiva Neonatal (UTIN), comparando-os às recomendações 
internacionais e avaliaram seu impacto sob a saúde do trabalhador; e um (2) identificou os fatores de risco psicossocial e a carga mental de trabalho de enfermeiros que trabalham em UTIN.

As informações inerentes aos agravos à saúde da equipe de enfermagem, fatores de riscos e sintomas associados à prática laboral no contexto neonatal constam na Tabela 3.

Tabela 3 - Distribuição dos agravos a saúde, fatores de riscos e sintomas relacionados à prática da enfermagem em UTIN, segundo as evidências analisadas nos 5 artigos. Goiânia, GO, 2020

\begin{tabular}{|c|c|c|c|}
\hline \multirow[b]{2}{*}{$\mathbf{N}^{\mathbf{0}}$} & \multicolumn{3}{|c|}{ Agravos a Saúde } \\
\hline & Dano Psicológico & Dano Físico & Dano Social \\
\hline & Fatores de risco/Sintomas & Fatores de risco/Sintomas & Fatores de risco/Sintomas \\
\hline 01 & $\begin{array}{c}\text { Estresse ocupacional/ } \\
\text { Baixa satisfação no trabalho }\end{array}$ & $\begin{array}{l}\text { Níveis constantes de ruído; } \\
\text { condições de trabalho } \\
\text { inadequadas/Fadiga }\end{array}$ & - \\
\hline 02 & $\begin{array}{l}\text { Desgaste emocional; } \\
\text { sobrecarga mental }\end{array}$ & $\begin{array}{l}\text { Jornada dupla de trabalho/ } \\
\text { Exaustão; } \\
\text { Enxaqueca; } \\
\text { Problemas gastrointestinais }\end{array}$ & $\begin{array}{c}\text { Irritabilidade; } \\
\text { Dificuldade de empatia; } \\
\text { Pouco apoio social e tempo para família } \\
\text { e atividades sociais }\end{array}$ \\
\hline 03 & $\begin{array}{l}\text { Aparecimento da Síndrome de } \\
\text { Burnout }\end{array}$ & $\begin{array}{l}\text { Elevados níveis de ruído/ Dores de } \\
\text { cabeça; } \\
\text { Fadiga; } \\
\text { Estresse; }\end{array}$ & - \\
\hline 04 & - & $\begin{array}{c}\text { Dificuldade de percepção } \\
\text { Elevado nível de ruído/ } \\
\text { Dificuldade de concentração }\end{array}$ & - \\
\hline 05 & $\begin{array}{l}\text { Medo; } \\
\text { Tristeza; } \\
\text { Impotência; } \\
\text { Raiva }\end{array}$ & $\begin{array}{l}\text { Carga de trabalho elevada/ } \\
\text { Transtornos musculoesqueléticos }\end{array}$ & $\begin{array}{l}\text { Dificuldades nas relações intra e } \\
\text { interprofissional; } \\
\text { Conflitos sociais; } \\
\text { Irritabilidade. }\end{array}$ \\
\hline
\end{tabular}

Fonte: Os autores.

A partir dos indicadores, expostos na tabela 3, emergiram duas categorias, a saber: fatores de riscos que predispõem ao agravo ocupacional na área neonatal e as principais consequências relacionadas à ocorrência dos agravos no ambiente laboral, que nortearam a discussão e reflexão desse estudo.

\section{Fatores de riscos que predispõem o agravo ocupacional na área neonatal}

A palavra dano tem origem no latim "dagnum" e é utilizada para descrever o mal ou o prejuízo que sofre uma pessoa ou um objeto por outrem (Bueno, 1996). O trabalho, a depender das condições em que é executado, é responsável por ocasionar danos à saúde dos trabalhadores, levando ao aparecimento de doenças, quer sejam de ordem física, psíquica ou emocional (Maciel, Santos \& Rodrigues, 2015; Oliveira \& Pereira, 2012).

Os danos atrelados ao trabalho representam a manifestação dos efeitos nocivos da atividade laboral para a saúde, em decorrência das intensas transformações no mundo do trabalho, que fazem emergir novos paradigmas entre o que é exigido e o que é oferecido por parte das instituições, contribuindo sobremaneira para o desequilíbrio físico, mental e social dos trabalhadores (Prestes et al., 2016).

Os danos físicos são definidos pela presença de manifestações como dores no corpo e distúrbios biológicos. Os danos psicológicos são caracterizados pela vivência de sentimentos negativos em relação a si mesmo e à vida no geral, enquanto os danos sociais compreendem o isolamento e dificuldades nas relações sociais e familiares (Mendes, 2007). 
Estes danos mostram-se evidentes nos diversos espaços laborais, todavia destaca-se que no âmbito hospitalar, e mais particularmente dentro do contexto neonatal tornam-se ainda mais relevantes, haja vista que à insalubridade, soma-se a convivência rotineira com o limiar entre vida e morte, alta complexidade, tecnologia avançada e agilidade no atendimento (Oliveira \& Sanino, 2011; Pedroza, Campos \& Oliveira, 2006).

Tais características, intrínsecas à realidade das unidades neonatal, são apontadas como alguns dos fatores responsáveis por predispor os trabalhadores ao prejuízo ocupacional, gerando desconforto, desgaste físico e conflitos emocionais (Fogaça et al., 2008).

Dentre os trabalhadores que estão inseridos nos espaços de trabalho da área da saúde, a equipe de enfermagem tem maior representatividade em relação as demais profissões (Carvalho et al., 2017). Estes trabalhadores, encontram-se em contato direto com os pacientes, durante 24 horas por dia, o que lhes conferem maior exposição à várias situações de risco. Além de tornarem-se mais vulneráveis aos agravos que comprometem, tanto, a sua saúde física, quanto emocional e mental (Fonsêca et al., 2016).

Nesse sentido, considera-se que os trabalhadores de enfermagem que cuidam de outros indivíduos, muitas vezes, se esquecem de cuidar de si próprios, e, do ambiente laboral. Esse esquecimento favorece o adoecimento do trabalhador, pelas condições pessoais e pelos ambientais desfavoráveis as atividades cotidianas (Ribeiro et al., 2012).

Face a isto, dois dos estudos ponderam que a presença de equipamento tecnológico complexo, níveis de ruído constantes dos muitos alarmes e atenção precisa aos detalhes necessários, estão dentre os elementos organizativos que potencializam a ocorrência do dano ocupacional em unidades neonatal (Curtis et al., 2015; Disher et al., 2017).

Sob esta perspectiva, particularmente, um dos estudos analisados destacou, ainda, que se tem aumentado a preocupação quanto aos níveis elevados de ruído nesses espaços de trabalho da enfermagem. Tal preocupação reside no fato do ruído ser um risco para as doenças profissionais que atinge o maior número de trabalhadores da enfermagem, especialmente quando associada ao aparecimento da Síndrome de Burnout, sendo sua intensidade diretamente proporcional ao dano ocasionado ao aparelho auditivo. Além de impactar consideravelmente no processo de trabalho dos trabalhadores que atuam em unidade neonatal (Galindo, Caicedo \& Vélez-Pereira, 2015).

Estudos têm apontado que a presença desses ruídos advém, especialmente, dos variados tipos de alarmes integrados aos modernos equipamentos, conversas altas e risadas, chamadas telefônicas dentro da unidade e movimentação de pessoas (Costa, Lacerda \& Marques, 2013; Pedroza, Campos \& Oliveira, 2006).

Outros aspectos associados às condições de trabalho têm sido descritos como preditores de danos à equipe de enfermagem neonatal. Dentre os quais, incluem-se: insuficiência de recursos humanos e materiais para prestação de cuidados; ritmo intenso de trabalho; conflito de papeis; sobrecarga de trabalho; jornadas de trabalho exaustivas; não reconhecimento profissional e baixa remuneração salarial (Barros \& Rodrigues, 2016; Ceballos-Vásquez et al., 2015; Maciel, Santos \& Rodrigues, 2015; Oliveira et al., 2013; Rizo \& Ubago, 2018; Terra et al., 2011).

Ademais, soma-se a isto a superlotação das unidades; elevada rotatividade; baixos índices de luminosidade em ambientes onde as atividades laborais são exercidas, assim como, padrões incorretos de temperatura e umidade relativa do ar (Almeida, Beltrame Junior \& Salazar, 2014).

Ante a existência desses elementos e a iminência do dano, emergem-se uma série de consequências que impactam a saúde dos trabalhadores inseridos nesses espaços laborais. 


\section{Principais consequências relacionadas à ocorrência de agravos à equipe de enfermagem neonatal no ambiente laboral}

Os danos decorrentes da vivência cotidiana dos trabalhadores no âmbito neonatal resultam em agravos que atingem não apenas o campo físico, como também, psicológico e social, dos trabalhadores, com destaque para os da equipe de enfermagem (Aytekin, Yilmaz\& Kuguoglu, 2013; Felli, 2012; Pedroza, Campos \& Oliveira, 2006).

Os diferentes métodos da organização do trabalho, associados às características peculiares das unidades neonatais, constituem fontes de pressão para os trabalhadores no exercício de suas atividades, o que acaba por intensificar o desgaste físico e psicológico do trabalhador. Em consequência, tem-se o fator desencadeante de estresse e sofrimento mental, contribuindo para o aparecimento de transtornos, como ansiedade, depressão e somatização de doenças (Oliveira \& Pereira, 2012; Oliveira et al., 2013).

Desta forma, foi consenso entre os estudos analisados que o dano psicológico se reflete nos sintomas relacionados ao estresse ocupacional, causado por exaustão do trabalhador e falta de satisfação pessoal, percussores da síndrome de Burnout (SB) (Ceballos-Vásquez et al., 2015; Galindo, Caicedo \& Vélez-Pereira, 2015).

Verificou- se, portanto, a presença de sentimentos como: raiva, culpa, vergonha, depressão, ansiedade, inadequação, insegurança e impotência (Curtis et al., 2015; Rizo \& Ubago, 2018). Corroborando outros estudos que destacam ainda: diminuição da concentração e memória, indecisão, confusão, perda do senso de humor, nervosismo, frustração, preocupação, medo, irritabilidade e impaciência (Felli, 2012; Lopes, Ribeiro \& Martinho, 2012; Oliveira, Tristão \& Neiva, 2006).

Ademais, destaca-se que, na maioria das vezes, o estresse ocupacional tem impacto de ordem coletiva na vida dos trabalhadores da enfermagem, concretizando o dano social. Assim, é capaz de influenciar de forma negativa os relacionamentos interpessoais e a capacidade profissional, criando, desta forma, um círculo vicioso (Oliveira et al., 2013; Silva et al., 2015).

Nesse interim, a Síndrome de Burnout tem sido majoritariamente identificada como um fenômeno psicossocial altamente associado à exaustão emocional e evidenciada por sentimentos de fadiga, esgotamento físico e emocional; pela despersonalização, em que o indivíduo se distancia das relações interpessoais; e pela baixa realização profissional, em que o profissional supre sentimentos negativos de si mesmo (Araújo et al., 2019; Maslach, 2009; Vitorino et al., 2018).

Estudos epidemiológicos têm apontado prevalências de Burnout em enfermeiros que variam entre 10\% a $29 \%$ (Quintas et al., 2017), chegando até mesmo ao índice de 43\% a 50\% (Albendin et al., 2016; Creedy et al., 2017; Lin et al., 2016; Trbojevic-Stankovic et al., 2015), estando o burnout correlacionado com a depressão, ansiedade, stress e isolamento social.

Os trabalhadores defrontam-se, portanto, com a dificuldade em expressar empatia pelos pacientes, familiares e demais membros da equipe, o que acaba por comprometer a eficácia e qualidade do cuidado, além de prejudicar as relações no ambiente de trabalho (Braithwaite, 2008).

Pode ainda gerar respostas desfavoráveis ao ambiente de trabalho e à instituição, uma vez que levam ao aumento das taxas de absenteísmo ou de presenteísmo, bem como de acidentes laborais e de afastamentos, com redução da produtividade (Carlotto, 2013; Pinto \& Chambel, 2008).

O isolamento social, fruto dessa cascata de sintomas, são extensivos aos relacionamentos fora do ambiente de trabalho, alterando o convívio social e familiar do trabalhador, em consequência do déficit na comunicação e hostilidade por parte desses trabalhadores (Batista et al., 2013; Graziano, Ferraz \& Bianchi, 2010).

Em se tratando dos danos físicos, os estudos analisados salientam a ocorrência de problemas gastrointestinais, hipertensão, doenças cardiovasculares, insônia, fadiga, dores de cabeça, tensão muscular e diminuição da função imune 
(Ceballos-Vásquez et al., 2015; Rizo \& Ubago, 2018). Incluem-se ainda: dores no corpo, palpitações, tremores, perda do apetite, pesadelos, sentimentos de inutilidade, extremidades frias e resfriados constantes (Dalmolin et al., 2012; Oliveira, Tristão \& Neiva, 2006).

Ressalta-se que, as manifestações físicas do dano estão relacionadas às principais causas e notificações de afastamentos do trabalho, cuja consequência manifesta-se em aposentadorias precoces e aumento dos índices de absenteísmos (Castro, Bernardino \& Ribeiro, 2008; Carvalho et al., 2017; Furlan \& Stancto, 2014; Gonçalves et al., 2005)

Considerado um problema administrativo, o absenteísmo gera um grave impacto dentro das instituições de saúde, especialmente nos setores que requerem mão de obra especializada, tal como, as unidades neonatais. Prediz, portanto, elevação dos custos para a contratação de novos trabalhadores, além de aumentar a sobrecarrega daqueles que permanecem inseridos nesses setores, potencializando e ou intensificando agravos à saúde (Martinato et al., 2010; Furlan \& Stancato, 2014).

Sob esse prisma, elucida-se a importância de um ambiente social acolhedor no controle do estresse ocupacional, tendo a organização um papel fundamental na mediação das variáveis que o predispõe. Assim, é fundamental que a instituição reconheça os estressores do ambiente na tentativa de estabelecer medidas que possam amenizar o sofrimento ou oferecer estratégias de apoio à equipe, tais como: fortalecimento da comunicação multiprofissional; discussões de dilemas éticos; capacitações quanto ao uso das tecnologias; maneiras de lidar com experiências de vida-morte bem como estímulo às atividades de lazer, religiosas e sociais (Oliveira, Xavier \& Araújo, 2013; Tito et al., 2013)

Salienta-se, portanto, que a prevenção dos riscos psicossociais e a criação de um ambiente psicossocial positivo nas organizações geram efeitos que sobrevêm de maneira global, não apenas sob os trabalhadores como também sob a própria instituição, dado que promovem o bem-estar físico e mental dos trabalhadores e melhora a sua satisfação com o trabalho, permitindo às instituições empregadoras beneficiar-se da melhoria global do desempenho profissional dos trabalhadores, do aumento dos níveis de motivação, eficácia e produtividade, assim como da redução do absentismo, do presenteísmo e das taxas de acidentes laborais, fato que se revela também altamente vantajoso pela redução de custos e encargos para a sociedade em geral (Quintas et al., 2017).

Considera-se, desta forma, que a saúde física e o bem-estar psicólogico dos enfermeiros são cruciais, pois deles dependem a segurança dos utentes e a qualidade dos cuidados que lhes são prestados, existindo, face a isto, um crescente reconhecimento da necessidade de reduzir-se ou mesmo evitar-se os danos ocupacionais no espaço de trabalho da enfermagem, pelas suas implicações altamente nefastas ou até mesmo fatais para todos aqueles envolvidos na assistência (Buunk, Zurriaga \& Peiró, 2010).

As evidências abarcadas nesse estudo permitiram mapear a realidade dos danos ocupacionais que sobrevém sob a saúde dos trabalhadores de enfermagem inseridos em unidades neonatal, e demonstraram a necessidade de robustecer as investigações na temática, visando a nortear políticas públicas na área da saúde do trabalhador, tendo em vista as limitações desta revisão, que incluem, a restrição das bases de dados e disponibilidade dos estudos na íntegra e idioma inglês, português e espanhol. Essa restrição impossibilita o alcance de alguns estudos de interesse para a pesquisa.

\section{Considerações Finais}

A pesquisa trouxe fundamentação teórica de vários autores internacionais acerca dos fatores de risco ocupacionais, sobre os quais ganharam destaque os físicos, psicológicos e sociais. Esses podem causar danos atrelados ao processo laboral e afetam majoritariamente a saúde dos trabalhadores de enfermagem em unidades neonatais.

As abordagens teóricas evidenciadas nos estudos permitiram concluir que os danos em unidade neonatal que atingem a equipe de enfermagem, associam-se, principalmente, à precariedade nas condições de trabalho que ao aliar-se às características próprias desses espaços, fazem emergir o estresse ocupacional. 
Sob esta ótica, as principais consequências observadas foram: sofrimento mental, e, por conseguinte, aparecimento de transtornos como ansiedade, depressão e somatização. Problemas nos sistemas osteoarticular, tecido muscular, trato digestivo, distúrbios osteomusculares, dor musculoesquelética, dor nas costas, isolamento social, dificuldades de relacionamento interpessoal e absenteísmo também caracterizaram resultados dos danos.

Diante dos resultados e por considerar que o sucesso e o bom desempenho das instituições de saúde dependem, dentre outros fatores, de trabalhadores saudáveis e motivados para o trabalho, recomenda-se a adoção de estratégias de prevenção aos agravos laborais, com medidas planejadas e executadas de forma transdisciplinar, considerando o trabalhador como um sujeito ativo, participativo e agente nesse processo, com vistas a uma política de saúde e segurança no trabalho, direcionadas ao preparo físico e psíquico dos trabalhadores de unidades neonatais, bem como estabelecimento de espaços de diálogos, resultando em melhoria da qualidade de vida e da assistência prestada.

Ressalta-se que não foi encontrado nenhum estudo nacional nesta revisão. Uma hipótese é que os danos atrelados ao trabalho da equipe de enfermagem, particularmente, em unidades neonatais seja um campo pouco explorado pelos pesquisadores brasileiros, o que é um fator alarmante, dado o impacto que sua ocorrência prevê sob a saúde dos profissionais aí atuantes.

Nesse ínterim, sugere-se a produção de novas pesquisas, especialmente no âmbito nacional, cujos achados poderão ser aplicados à prática assistencial, direcionando, assim, para a promoção do bem-estar físico-psíquico-social da equipe de enfermagem em seu espaço de trabalho.

É preciso, portanto, incentivar o uso da lente que prisma por um ambiente hígido, fomentando, uma gestão organizacional focada no processo de trabalho e no ambiente, e com isso, novos constructos no que diz respeito à uma cultura de segurança do trabalhador na instituição de saúde.

\section{Referências}

Albendin L. et al. (2016). Prevalencia bayesiana y niveles de burnout en enfermeria de urgências. Revista Latino Americana de Psicologia, Espanha, n. 48, p. 137-145, nov. 2016.

Agostini M. (2002). Saúde do Trabalhador. In: Andrade A; Pinto S C; Oliveira RS. (orgs). Animais de Laboratório: criação e experimentação [online]. Rio de Janeiro: Fiocruz, 2002. 388 p.

Almeida L M N; Beltrame Júnior M; \& Salazar M B P. (2014). Unidade de Terapia Intensiva Neonatal: riscos físicos como fatores potenciais de agravos à saúde do trabalhador. Revista Univap, São Paulo, v. 20, n. 35, p. 69-77, 2014.

Araújo A L B. et al. (2019). Síndrome de Burnout em Enfermeiros que atuam em Unidade de Terapia Intensiva Neonatal. Motricidade, Santa Maria da Feira, v. 15 , n. 4 , p. 51-58, 2019.

Aytekin A; Yilmaz F; \& Kuguoglu S. (2013). Burnout levels in neonatal intensive care nurses and its effects on their quality of life. Australian Journal of Advanced Nursing, Austrália, v. 31, n. 2, p. 39, 2013.

Barros A R; \& Rodrigues L M. (2016). O exercício profissional de enfermagem e as principais causas de adoecimento laboral: uma revisão integrativa. Revista Científica de Enfermagem, São Paulo, v. 6, n. 18, p. 12-25, 2016.

Batista J B V. et al. (2013). Síndrome de Burnout: compreensão de profissionais de enfermagem. Revista de enfermagem UFPE on line, Pernambuco, v. 7, n. 2 , p. 553-561, 2013.

Bendett A; Ferraz L; \& Posso M B S. (2009). Riscos para agravos musculoesqueléticos entre os profissionais de saúde de uma unidade de terapia intensiva neonatal. Revista da Sociedade Brasileira de Enfermeiros Pediatras, São Paulo, v. 9, n.2, p. 59-65, 2009.

Braithwaite M. (2008). Nurse burnout and stress in the NICU. Advances in neonatal care, Philadelphia, v. 8, n. 6, p. 343-347, 2008.

Bueno F S. (1996). Minidicionário da língua portuguesa. São Paulo: FTD LISA, 1996.

Buunk A P; Zurriaga R; \& Peiró J m. (2010). Social comparison as a predictor of changes in burnout among nurses. Anxiety, Stress \& Coping, Londres, v. 23, n. 2, p. 181-194, 2010.

Carvalho D P et al. (2017). Cargas de trabalho e a saúde do trabalhador de enfermagem: revisão integrativa. Cogitare Enfermagem, Paraná, v. 22 , n. 1, 2017.

Castro I; Bernardino E; \& Ribeiro E L Z. (2008). Absenteísmo na enfermagem em UTI neonatal: perfil do profissional e motivos das ausências. Cogitare Enfermagem, Curitiba, v.13, n.3, 2008. 
Cavaliere, T.A. et al. (2010). Moral distress in neonatal intensive care unit RNs. Advances in Neonatal Care, Philadelphia, v. 10, n. 3, p.145-156, 2010.

Ceballos-Vásquez, P. et al. (2015). Fatores psicossociais e carga mental de trabalho: uma realidade percebida pelos enfermeiros em Unidades de Terapia Intensiva. Revista Latino-Americana De Enfermagem, Ribeirão Preto, v. 23, n. 2, p. 315-322, 2015.

Costa, D. et al. (2013). Saúde do Trabalhador no SUS: desafios para uma política pública. Revista Brasileira de Saúde Ocupacional, São Paulo, v. 38, n. 127, p.11-21, 2013

Costa, G. L; Lacerda, A.B.M; \& Marques, J. (2013). Ruído no contexto hospitalar: impacto na saúde dos profissionais de enfermagem. Revista CEFAC, Campinas, v. 15, n. 3, p. 642-652, 2013.

Creedy, D. K. et al. (2017). Prevalence of burnout, depression, anxiety and stress in Australian midwives: a cross-sectional survey. BMC Pregnancy and Childbirth, Londres, v. 17, n. 13, p. 1-8, 2017.

Curtis, Dc. et al. (2015). News of the Academy of Neonatal. Academy of Neonatal Nursing, Estados Unidos, v. 34, n.5, p. 300-307, 2015.

Dalmolin, G. L. et al. (2012). Implicações do sofrimento moral para os (as) enfermeiros (as) e aproximações com o Burnout. Texto \& Contexto Enfermagem, Florianópolis, v. 21, n. 1, p. 200-208, 2012.

Dejours, C; Abdoucheli, E; \& Jayet, C. (2014). Psicodinâmica do trabalho: contribuições da escola Dejouriana à análise da relação prazer, sofrimento e trabalho. São Paulo: Atlas, 2014.

Disher, Tc. et al. (2017). Striving for optimum noise-decreasing strategies in critical care. The Journal of perinatal \& neonatal nursing, Frederick MD, v. 31, n. 1, p. 58-66, 2017.

Ercole, Ff; Melo, Ls ; \& Alcoforado, C 1. G .C. (2014). Revisão integrativa versus revisão sistemática. Revista Mineira de Enfermagem, Minas Gerais, v. 18, n. 1, p.9-12, 2014

Felli, V.E.A. (2012). Condições de trabalho de enfermagem e adoecimento: motivos para a redução da jornada de trabalho para 30 horas. Enfermagem em foco, Brasília, v. 3, n. 4, p. 178-181, 2012.

Fogaça, M.C. et al. (2010). Fatores que tornam estressante o trabalho de médicos e enfermeiros em terapia intensiva pediátrica e neonatal: estudo de revisão bibliográfica. Revista Brasileira de Terapia Intensiva, Rio de Janeiro, v. 20, n. 3, p. 261-266, 2010.

Fonsêca, L.D.C.T.D. et al. (2016). Vulnerability of the health of nursing professionals acting in the hospital setting: integrative review. Revista de enfermagem UFPE on line, Pernambuco, v. 10, n. 7, p. 2687-2695, 2016.

Forte, E.C.N. et al. (2014). Abordagens teóricas sobre a saúde do trabalhador de enfermagem: revisão integrativa. Cogitare Enfermagem, Curitiba, v. 19, n. 3, p. 304-611, 2014

Furlan, J.A.D.S; (2013). Stancato, K. Fatores geradores do absenteísmo dos profissionais de enfermagem de um hospital público e um privado. Revista de Administração em Saúde, São Paulo, v. 15, n. 60, p. 111-120, 2013.

Galindo, Apg; Caicedo, Yc; \& Vélez-Pereira, Am. (2015). Nivel continuo equivalente de ruido en la unidad de cuidado intensivo neonatal asociado al síndrome de burnout. Enfermería Intensiva, Madrid, v. 26, n. 3, p. 92-100, 2015.

Gonçalves, J. R. D. S. et al. (2005). Causas de afastamento entre trabalhadores de enfermagem de um hospital público do interior de São Paulo. Revista Mineira de Enfermagem, Minas Gerais, v. 9, n. 4, p. 309-314, 2005.

Grazziano, E.S; Ferraz \& Bianchi, E.R. (2010). Impacto del estrés ocupacional y burnout en enfermeros. Enfermería Global, Murcia, n. 18, p.1-20, 2010.

Karino, M. E; Martins, Jt; \& Bobroff, M. C. C. (2011). Reflexão sobre as políticas de saúde do trabalhador no brasil: avanços e desafios. Ciência, Cuidado e Saúde, Maringá, v. 10, n. 2, p. 395-400, 2011.

Lin, Tc. et al. (2016). Work stress, occupational burnout and depression levels: a clinical study of pediatric intensive care unit nurses in Taiwan. Journal of Clinical Nursing, Oxford, n. 25, p. 1120-1130, 2016.

Lopes, C.C.P; Ribeiro, T.P; \& Martinho, N.J. (2012). Síndrome de Burnout e sua relação com a ausência de qualidade de vida no trabalho do enfermeiro. Enfermagem em Foco, Brasília, v. 3, n. 2, p. 97-101, 2012.

Maciel, R.H.M.O; Santos, J.B.F; \& Rodrigues, R.L. (2015). Condições de trabalho dos trabalhadores da saúde: um enfoque sobre os técnicos e auxiliares de nível médio. Revista Brasileira de Saúde Ocupacional, São Paulo, v. 40, n. 131, p. 75-87, 2015.

Martinato, M.C.N.B. et al. (2011). Absenteísmo na enfermagem: uma revisão integrativa. Revista Gaúcha de Enfermagem, Porto Alegre, v. 31, n.1, p.160$166,2010$.

Masson, Lp; Brito, J; \& Athayde, M. (2018). Dimensão relacional da atividade de cuidado e condições de trabalho de auxiliares de enfermagem em uma unidade neonatal. Physis, Rio de Janeiro, v. 21, n. 3, p. 879-898, 2011.

Melnyk, B. M; \& Fineout-Overholt, E. (2005). Making the case for evidence-based practice. In: MELNYK, BM; FINEOUT-OVERHOLT, E (orgs). Evidence based practice in nursing \& healthcare. A guide to best practice. Philadelphia: Lippincot Williams \& Wilkins, 2005. p. 3-24.

Mendes, A.M. (2007). Psicodinâmica do trabalho: teoria, método e pesquisas. São Paulo: Casa do Psicólogo, 2007. 
Mendes, K.D.S; Silveira, R.C.P; \& Galvão, C.M. (2008). Revisão integrativa: método de pesquisa para a incorporação de evidências na saúde e na enfermagem. Texto \& Contexto Enfermagem, Florianópolis, v. 17, n. 4, p.758-764, 2008.

Milette, I. (2010). Decreasing noise level in our NICU: the impact of a noise awareness educational program. Advances in Neonatal Care, Philadelphia, v. 10, n. 6, p.343-351, 2010 .

Nunes, B. K; \& Toma, E. (2013). Dimensionamento de pessoal de enfermagem de uma unidade neonatal: utilização do Nursing Activities Score. Revista Latino-Americana de Enfermagem, Ribeirão Preto, v. 21, n.1, p.348-355, 2013.

Oliveira, D. C; Xavier, J. L; \& Araújo, L. G. S. (2013). The disease process nursing. Revista de Enfermagem da UFPI, Teresina, v. 2 , n. spe, p.76-9, 2013.

Oliveira, E .B. D. et al. (2013). Fatores de risco psicossocial em terapia intensiva neonatal: repercussões para a saúde do enfermeiro. Revista de Enfermagem UERJ, Rio de Janeiro, v. 21, n. 4, p. 490-495, 2013.

Oliveira, L. L; \& Sanino, G. E. C. (2011). A humanização da equipe de enfermagem em unidade de terapia intensiva neonatal: concepção, aplicabilidade e interferência na assistência humanizada. Revista da Sociedade Brasileira de Enfermeiros Pediatras, São Paulo, v. 11, n. 2, p.75-83, 2011.

Oliveira, P. R; Tristão, R. M; \& Neiva, E.R . (2006). Burnout e suporte organizacional em profissionais de UTI-Neonatal. Educação Profissional: Ciência e Tecnologia, Brasília, v. 1, n. 1, p.27-37, 2006.

Oliveira, V; \& Pereira, T. (2012). Ansiedade, depressão e burnout em enfermeiros: Impacto do trabalho por turnos. Revista de Enfermagem Referência, Coimbra, v. serIII, n. 7, p. 43-54, 2012.

Pedroza, M. A. D; Campos, A. C. S; \& Oliveira, M. M. (2006). Dano ocupacional na unidade de terapia intensiva neonatal: a percepção da enfermeira. Revista da Rede de Enfermagem do Nordeste, Ceará, v. 7, n.2. p. 17-24, 2006.

Pires, D. E. P de. et al. (2012). Inovação tecnológica e cargas de trabalho dos profissionais de saúde: uma relação ambígua. Revista Gaúcha de Enfermagem, Porto Alegre, [s.1.], v. 33, n. 1, p.157-168, mar. 2012.

Prestes, F. C. et al. (2016). Danos à saúde dos trabalhadores de enfermagem em um serviço de hemodiálise. Revista Gaúcha de Enfermagem, Porto Alegre, v. 32, n. 1, e50759, 2016.

Quintas, S. et al. (2017). Os enfermeiros e a sua saúde no trabalho: a relação entre depressão e burnout. International Journal on Working Conditions, Porto, n. 13 , p. 1-20, 2017.

Ribeiro, E; \& Shimizu, H. E. (2020). Acidentes de trabalho com trabalhadores de enfermagem. Revista Brasileira de Enfermagem, Brasília, v. 60, n. 5, p. 535540, 2007. Disponível em: http://www.scielo.br/scielo.php?script=sci_arttext\&pid=S0034-71672007000500010\&lng=en. Acesso em: 19 out. 2020.

Ribeiro, R. P. et al. (2012). O adoecer pelo trabalho na enfermagem: uma revisão integrativa. Revista da Escola de Enfermagem da USP, São Paulo, v. 46, n. 2, p. 495-504, 2012.

Rizo, M 1 p; \& Ubago, M v. (2018). Estudio descriptivo sobre las condiciones de trabajo y los trastornos musculo esqueléticos en el personal de enfermería (enfermeras y AAEE) de la Unidad de Cuidados Intensivos Pediátricos y Neonatales en el Hospital Clínico Universitario de Valladolid. Medicina y Seguridad del Trabajo, Madrid, v. 64, n. 251, p. 161-199, 2018.

Rolim, K. M. C; Oliveira, M. M. C; \& Cardoso, M. V. L. M. L. (2003). Combate ao estresse na Unidade de Internação Neonatal: uma experiência grupal. Revista da Rede de Enfermagem do Nordeste, Ceará, v. 4, n. 1, 2003.

Silva, J. L. L. et al. (2015). Fatores psicossociais e prevalência da síndrome de burnout entre trabalhadores de enfermagem intensivistas. Revista Brasileira de Terapia Intensiva, Rio de Janeiro, v. 27, n. 2, p. 125-133, 2015.

Souza, M. T; Silva, M. D; \& Carvalho, R. I (2010). ntegrative review: what is it? How to do it? Einstein, São Paulo, v. 8, n. 1, p. 102-106, 2010.

Terra, A. A. A. et al. (2011). O processo de trabalho da enfermagem em unidade de terapia intensiva neonatal. HU Revista, Juiz de Fora, v. 37 , n. 1, 2011.

Tito, R. S. et al. (2015). Síndrome de Burnout em enfermagem pediátrica e neonatal: revisão da literatura. Enfermagem em Foco, Brasília, v. 4, n. 3/4, p.194$197,2015$.

Trbojevic-Stankovic, J. et al. (2015). Work-related factors as predictors of burnout in Serbian nurses working in hemodialysis. Nephrology Nursing Journal, Pitman, v. 42, n. 6, p. 553-562, 2015.

Ursi, E. S. (2018). Prevenção de lesões de pele no perioperatório: revisão integrativa da literatura. 2005. 130p. Dissertação (Mestrado em Enfermagem) Escola de Enfermagem de Ribeirão Preto, Universidade de São Paulo, São Paulo, 2005.

Vitorino, M.F. et al. Síndrome de burnout: conhecimento da equipe de enfermagem neonatal. Revista de enfermagem UFPE on line, Pernambuco, v. 12, n. 9, p. 2308-2314, 2018. 